\title{
Serial Block Face Imaging of Aluminium
}

\author{
Joel Mancuso $^{1}$, Teruo Hashimoto ${ }^{2}$, George Thompson ${ }^{2}$ \\ ${ }^{1}$ Gatan Inc., Pleasanton, CA. \\ ${ }^{2}$ The University of Manchester, School of Materials, Corrosion \& Protection Centre, Oxford Road, \\ Manchester, M13 9PL, U.K.
}

Three dimensional data acquisition techniques are allowing researchers to ask new questions. An essential question is how three-dimensional geometry of materials organized. Researchers have several options at the Transmission Electron Microscope (TEM) level including, tomography, and serial section imaging. Although tomography has excellent resolution, the field of view is limited and it is impractical for large volume acquisitions. Serial sectioning is used for obtaining large volume 3D electron microscopic data, but the technique is a highly skilled time consuming process prone to human error. Serial block face scanning electron microscopy (SBFSEM) is a new automated technique obtaining serial images using a scanning electron microscope (SEM). Bridging the gap between ultrahigh resolution tomography, and light microscopy, SBFSEM allows a streamlined and automated 3D data acquisition process. A microtome equipped with a diamond knife is mounted inside the chamber of the SEM and shaves off 50nm of the sample in between imaging. Images are collected using a back scatter (BS) electron detector, which result in a classic TEM like image. The SBFSEM imaging process is completely automated allowing for large volume acquisition in a timely manner.

We have used SFBSEM insitu microtome to cut a variety of materials. Until recently SBFSEM has primarily been a life science imaging technique. Using aluminium, we demonstrate acquiring large volume high resolution data in the field of material science. In addition we will demonstrate joining SBFSEM with other techniques such as X-ray elemental analysis, creating 3D elemental maps. Using SBFSEM as a life science tool obtaining slices below $30 \mathrm{~nm}$ has been a limitation mainly due to the epoxy resin that biological samples are embedded in. We will demonstrate 3 Views ability to cut aluminium well below this limitation resulting is isotropic voxels below $10 \mathrm{~nm}$.

Figure A. SBFSEM image of aluminium with cracked in inclusion of copper and magnesium. Original image size of $2048 \times 2048$ with $14 \mathrm{~nm}$ pixels: over 300 serial images were acquired.

Figure B. Volume reconstruction using 3D visualization packed in DigitalMIcrograph, of the previous data set.

Figure C. Serial images taken from every fifth slice, images are $200 \mathrm{~nm}$ apart showing cracked inclusions in aluminium. 
